'Laboratorio de Nefrotoxicidad y Estrés Oxidativo, Programa de Farmacología Molecular y Clínica, Facultad de Medicina. Universidad de Chile. Santiago, Chile.

${ }^{2}$ Laboratorio de Ciencias de la Actividad Física, Deporte y la Salud; Facultad de Ciencias Médicas. Universidad de Santiago de Chile. Santiago, Chile. ${ }^{3}$ Escuela de Educación Física, Facultad de Educación, Universidad de las Américas UDLA. Santiago, Chile

${ }^{4}$ Escuela de Educación Física, Facultad de Educación, Universidad Autónoma de Chile, Santiago, Chile 5Laboratorio de Inmunología de la Reproducción, Facultad de Química y Biología. Universidad de Santiago de Chile. Santiago, Chile. ${ }^{6}$ Escuela de Ciencias del Deporte. Facultad de Salud. Universidad Santo Tomás, UST Chile

aTerapeuta en Actividad Física y Salud, Profesor de Educación Física, MSc en Fisiología. bProfesor de Educación Física, MSc en Fisiología.

'Químico Farmacéutico, $\mathrm{PhD}{ }^{\odot}$ en Farmacología. dProfesor de Educación Física. 'Terapeuta en Actividad Física y Salud. fProfesor de Educación Física. PhD.

Los autores declaran no tener conflictos de interés. Trabajo no recibió financiamiento.

Recibido el 4 de junio de 2017, aceptado el 3 de diciembre de 2017

Correspondencia a: Carlos Poblete-Aro

Laboratorio de Nefrotoxicidad y Estrés Oxidativo, Programa de Farmacología Molecular y Clínica, Facultad de Medicina. Universidad de Chile. Santiago, Chile carlos.poblete.aro@gmail.com

\section{Efecto del ejercicio físico sobre marcadores de estrés oxidativo en pacientes con diabetes mellitus tipo 2}

\author{
CARLOS POBLETE-ARO ${ }^{1,2,3, a}$, JAVIER RUSSELL-GUZMÁN ${ }^{4, b}$, \\ PABLO PARRA ${ }^{1, \mathrm{c}}$, MARCELO SOTO-MUÑOZ ${ }^{2, \mathrm{~d}}$, \\ BASTIÁN VILLEGAS-GONZÁLEZ ${ }^{2, e}$, CRISTIÁN COFRÉ-BOLA- \\ DOS $^{2, \mathrm{f}}$, TOMÁS HERRERA-VALENZUELA ${ }^{2,5,6, \mathrm{f}}$

\section{Exercise and oxidative stress in type 2 diabetes mellitus}

Sedentarism, overweight and malnutrition generate an increase in the production of reactive oxygen species leading to a state of chronic oxidative stress. In patients with type 2 diabetes mellitus, oxidative stress alters pancreatic insulin secretion and the actions of the hormone on target cells, contributing to the development of micro and macrovascular complications. During physical exertion a state of transient oxidative stress occurs. As a consequence, the organism generates multiple physiological adaptations to these repetitive stimuli. Physical exercise is beneficial for type 2 diabetes mellitus but there is a paucity of information about the effects of physical exercise on biomarkers of oxidative stress in patients with the disease. We herein try to elucidate if the effects of exercise on oxidative stress can help in the prevention and treatment of type 2 diabetes mellitus and which is the most effective modality of physical exercise to reduce oxidative stress markers.

(Rev Med Chile 2018; 146: 362-372)

Key words: Antioxidant Response Element; Diabetes Mellitus, Type 2; Exercise; Oxidative Stress.
L a diabetes mellitus tipo 2 (DMT2) es una enfermedad crónica no transmisible con una alta prevalencia a nivel global ${ }^{1,2}$. Esta enfermedad se asocia a factores de riesgo modificables tales como el sedentarismo y malnutrición ${ }^{2}$, y se vincula fuertemente a enfermedades cardiovasculares y cáncer, las dos principales causas de muerte en el mundo ${ }^{3}$. En los últimos años se ha encontrado una relación entre los biomarcadores de estrés oxidativo y la DMT2 ${ }^{4}$. $\mathrm{Al}$ respecto se ha observado que el estrés oxidativo desempeña un papel de relevancia tanto en la patogénesis de la $\mathrm{DMT}^{5}$, así como también en el desarrollo de complicaciones asociadas a la enfermedad ${ }^{6}$.

La realización de ejercicio físico ha sido ampliamente recomendada debido a sus múltiples beneficios para la salud ${ }^{7,8}$. Resultados obtenidos a partir de distintas revisiones sistemáticas han mostrado que intervenciones de ejercicio físico reducen tanto estadística como clínicamente los niveles de hemoglobina glicosilada (HbAlc) en pacientes con DMT2 ${ }^{9,10}$. En paralelo, se ha obser- 
vado que intervenciones de ejercicio físico inducen una respuesta de adaptación caracterizada por la disminución de marcadores de daño por estrés oxidativo, así como también por un incremento de la respuesta antioxidante del organismo ${ }^{11}$. No obstante, también existe evidencia contradictoria respecto a la efectividad del ejercicio físico en la disminución de marcadores de estrés oxidativo y si dichos efectos pueden tener un papel de relevancia en la prevención y/o tratamiento de la patología. Por lo tanto, el objetivo de la presente revisión bibliográfica es describir los mecanismos de adaptación inducidos por el ejercicio físico y resumir el efecto de distintos protocolos de ejercicio físico sobre biomarcadores de estrés oxidativo, con el fin de discutir el potencial preventivo y terapéutico de la intervención y su vinculación con el control del balance redox en pacientes adultos diagnosticados con DMT2.

\section{Balance redox y estrés oxidativo}

En condiciones fisiológicas existe un estado de equilibrio en el balance redox resultante de la interacción funcional entre los agentes oxidantes derivados de la reducción incompleta del oxígeno en $\mathrm{H}_{2} \mathrm{O}$ llamadas especies reactivas del oxígeno $(\text { EROs })^{12,13}$, tales como anión superóxido $\left(\mathrm{O}_{2}{ }^{-}\right)$, radical hidroxilo $\left(\mathrm{OH}^{-}\right)$, peróxido de hidrógeno $\left(\mathrm{H}_{2} \mathrm{O}_{2}\right)^{12,13}$ y de agentes antioxidantes enzimáticos como superóxido dismutasa (SOD), catalasa (CAT), glutatión peroxidasa (GPx), y no-enzimáticos (ej. glutatión reducido, vitamina $\mathrm{C})^{12,13}$. No obstante, cuando se pierde el equilibrio del balance redox por una mayor generación de agentes oxidantes por sobre los agentes antioxidantes, se produce estrés oxidativo $(\mathrm{EO})^{13}$. El EO de carácter crónico es dañino para la célula, ya que provoca oxidación de macromoléculas y genera cambios en las vías de señalización celular por modificaciones en el balance redox, alterando la función celular, además de poder inducir su muerte ${ }^{11,14}$.

La evaluación del estado redox en el organismo es compleja debido a la existencia de una gran variedad de biomarcadores tanto para agentes oxidantes como para agentes antioxidantes, por lo que se recurre a establecer una relación entre ambas partes ${ }^{4}$. Las estrategias más utilizadas en humanos para evaluar el EO son: I) medir la abundancia y/o actividad de proteínas antioxidantes; II) cuantificar productos derivados de la oxidación y III) analizar el balance oxidante-antioxidante ${ }^{15,16}$.

Gran parte de estos biomarcadores se pueden detectar en muestras de sangre (eritrocitos, plasma) u orina. $\mathrm{Al}$ respecto, se ha observado una concordancia entre los niveles de biomarcadores de EO en sangre y en órganos centrales tales como el hígado, músculo esquelético, corazón y riñón ${ }^{16}$. Estos antecedentes sugieren que el análisis de biomarcadores de EO obtenidos a partir de muestras de sangre, pueden ofrecer una visión panorámica del estado redox del organismo ${ }^{16}$. En la Tabla 1 se resumen los principales marcadores de $\mathrm{EO}^{16}$.

\section{Estrés oxidativo y DMT2}

En pacientes con DMT2, existe una producción aumentada de EROs y una menor actividad antioxidante ${ }^{5}$. La disfunción mitocondrial y la desregulación de enzimas pro-oxidantes parecen ser los principales factores involucrados en la generación crónica de EROs induciendo un estado de EO crónico. A nivel de la mitocondria, la desregulación de proteínas relacionadas con los procesos de fisión y fusión provocan cambios morfológicos en las membranas interna y externa de este organelo, modificando el potencial de membrana mitocondrial, lo que conlleva a alteraciones en la función de la cadena transportadora de electrones y con esto una fuga de EROs desde los complejos I y III principalmente ${ }^{17}$. En relación a las enzimas pro-oxidantes, se ha observado un aumento en la actividad de la enzima NADPH oxidasa 2 (NOX2) en el músculo esquelético, y con ello, un aumento en la producción de EROs basal ${ }^{18}$. Ambos procesos contribuyen en la generación de resistencia a la insulina (RI) debido a una alteración en la función de sustratos del receptor de insulina (SRI), los cuales son sensibles a cambios en el balance redox ${ }^{19}$. De este modo, existe una alteración en la translocación de los transportadores de glucosa GLUT4 desde el citoplasma hacia la membrana celular $^{20,21}$. Posteriormente se genera disfunción de las células $\beta$ pancreáticas por una mayor secreción de insulina, con el fin de compensar la RI de los diferentes órganos afectados ${ }^{21,22}$.

En casos en que la DMT2 se encuentra vinculada a cuadros de sobrepeso/obesidad ${ }^{23}$, se observa un aumento en los niveles de biomarcadores de daño por EO inducida por infiltración de macró- 
Tabla 1. Resumen de marcadores de estrés oxidativo. Adaptado de Mañon $2016^{16}$

\begin{tabular}{|c|c|}
\hline Clasificación & Marcadores de estrés oxidativo \\
\hline \multicolumn{2}{|l|}{ Antioxidantes } \\
\hline \multirow[t]{2}{*}{ Endógenos } & $\begin{array}{l}\text { a) Enzimáticos } \\
\text { Superóxido dismutasa (SOD) } \\
\text { Glutatión peroxidasa (GPX) } \\
\text { Catalasa (CAT) } \\
\text { Glutatión reductasa (GR) } \\
\text { Tiorredoxina reductasa (TR) }\end{array}$ \\
\hline & $\begin{array}{l}\text { b) No enzimáticos: } \\
\text { Glutatión } \\
\text { Tiorredoxina } \\
\text { Peroxirredoxina } \\
\text { Bilirrubina } \\
\text { Coenzima Q10 } \\
\text { Urato }\end{array}$ \\
\hline Exógenos (dieta) & $\begin{array}{l}\text { Ácido } \alpha \text {-lipoico } \\
\beta \text {-caroteno } \\
\text { Vitamina E ( } \alpha \text {-tocoferol) } \\
\text { Vitamina C (ácido ascórbico) }\end{array}$ \\
\hline Otros marcadores & Capacidad antioxidante total \\
\hline \multicolumn{2}{|l|}{ Productos derivados de oxidación } \\
\hline Aminoácidos y proteínas & $\begin{array}{l}\text { Nitrotirosina } \\
\text { Proteínas carboniladas (PC) }\end{array}$ \\
\hline Lípidos & $\begin{array}{l}\text { Isoprostanos } \\
\text { 4-hidroxinonenal (4-HNE) } \\
\text { Sustancias reactivas al ácido tiobarbitúrico (TBARS) } \\
\text { Malondialdehído (MDA) } \\
\text { Palmitoil oxovaleroil fosforilcolina (POVPC) } \\
\text { Palmitoil glutaril fosforilcolina (PGPC) }\end{array}$ \\
\hline Ácidos nucleicos & 8-oxo-7,8-dihidro-2'-desoxiguanosina (8-OH-dG) \\
\hline Balance antioxidante/oxidante & $\begin{array}{l}\text { Relación GSH/GSSH } \\
\text { Estado redox de cisteína } \\
\text { Estado tiol/disulfido }\end{array}$ \\
\hline
\end{tabular}

Siglas: GSH: glutatión reducido; GSSH: glutatión oxidado.

fagos $M 1$ en el tejido adiposo ${ }^{22}$. Se ha propuesto que el EO generado por la disfunción mitocondrial y desregulación de NOX2 en el músculo esquelético, junto con la disfunción del adipocito en sobrepeso/obesidad inducida por macrófagos M1 juegan un papel relevante en la patogénesis de la DMT2 $2^{5,23}$. Teniendo en cuenta el papel que desempeña el EO en la patogénesis de la DMT2 y el desarrollo de complicaciones asociadas, la evidencia actual sugiere que la disminución de factores pro oxidantes o el aumento de factores antioxidantes debe ser considerado en el tratamiento de esta patología ${ }^{14,16}$.

\section{Estrés oxidativo inducido por ejercicio y hormesis}

La práctica regular de ejercicio físico ha sido ampliamente promovida debido a sus múltiples efectos para la salud, entre los cuales destacan el aumento de la función cardiovascular y respiratoria, mejora de la composición corporal, mayor control glicémico, entre otros ${ }^{8}$. En la última década, diversos reportes han indicado que la realización de ejercicio físico promueve la producción de EROs debido principalmente a una elevación de la actividad excito-contráctil del músculo es- 
quelético $^{11,25}$. Dicho fenómeno genera un desbalance redox de carácter transitorio denominado "estrés oxidativo inducido por ejercicio" ${ }^{15}$. Una de las principales fuentes de EROs en el músculo esquelético durante la realización de ejercicio es el complejo enzimático NOX2, localizado en la membrana del sarcolema, túbulo transversal y retículo sarcoplasmático del músculo esquelético ${ }^{15}$. Durante la despolarización de la fibra muscular existe liberación de ATP vía pannexina, proceso que permite la activación de receptores purinérgicos asociados a la vía de señalización de $\mathrm{IP}_{3}$, generando liberación de $\mathrm{Ca}^{2+}$ desde el retículo sarcoplasmático (RS) y activación de la proteínas kinasas dependientes de $\mathrm{Ca}^{2+}$ (PKC). La PKC fosforila la subunidad citoplasmática de NOX2 (p47/ Phox), generando un cambio conformacional que favorece el acoplamiento de esta a las subunidades de la membrana plasmática de NOX2, volviendo al complejo NOX2 funcional ${ }^{26}$. Otros mecanismos de producción de EROs en el músculo esquelético corresponden a la activación de enzimas como la fosfolipasa $\mathrm{A}_{2}\left(\mathrm{PLA}_{2}\right)$, óxido nítrico sintetasa neural (nNOS), y en menor medida, la generación de $\mathrm{O}_{2}{ }^{*}$ - desde la cadena transportadora de electrones mitocondrial ${ }^{15}$. En paralelo, el endotelio de los vasos sanguíneos que irrigan al tejido muscular también está involucrado en la producción de EROs, siendo las enzimas xantina oxidasa $(\mathrm{XO}) \mathrm{y}$ óxido nítrico sintasa endotelial (eNOS) las principales responsables ${ }^{15}$.

La producción de EROs durante la realización de ejercicio es aguda y de corta duración, pudiendo aumentar los niveles de biomarcadores de daño por EO como malondialdehído (MDA) y proteínas carbonilo (PC) en sangre entre 24 a $48 \mathrm{~h}^{27}$. Las EROs generadas durante la realización de ejercicio inducen adaptaciones siguiendo el principio de "hormesis"11, permitiendo activar distintas vías de señalización, las cuales a su vez confluyen en la activación de factores transcripcionales tales como los factores Nrf2, NF- $\kappa \mathrm{B}$ y el cofactor de transcripción PGC-1 $\alpha^{25}$.

Nrf2 normalmente está secuestrado en el citosol por Keap1. Tras la estimulación oxidativa, se modifican covalentemente los grupos tiol de Keap1 disociandose de Nrf2, permitiendo que éste transloque al núcleo uniéndose a los ARE (“Antioxidants Response Elements") que aumentan la expresión de enzimas antioxidantes ${ }^{28,29}$.

En el caso del complejo NF- $\kappa B$, las EROS di- socian el complejo trimérico preformado por la proteína inhibitoria I $\kappa$ B (inhibidor de NF- $\kappa \mathrm{B}$ ) y el dímero de proteína p50/p65. Al mismo tiempo, inducen cambios redox que dan como resultado la fosforilación de la subunidad I $\mathrm{B}$, activando de este modo su digestión proteolítica. Cuando la subunidad I $\kappa$ B se desprende del heterodímero p60/p65, NF- $\kappa B$ puede translocarse al núcleo, unirse a $\mathrm{ADN}$ e iniciar la transcripción asociadas a citokinas como IL-6 ${ }^{28,29}$.

PGC-1 $\alpha$ actúa como un coactivador transcripcional a través del reclutamiento y la corregulación de múltiples factores de transcripción que regulan la expresión génica del músculo esquelético, incluyendo Nrf-2, lo que permite la biogénesis mitocondrial junto con el aumento de la expresión de enzimas antioxidantes ${ }^{28,29}$.

La activación de Nrf2, NF- $\kappa \mathrm{B}$ y PGC- $1 \alpha$ inducida por el ejercicio permite el aumento de contenido y actividad de las enzimas SOD, CAT y $\mathrm{GPx}^{25-27,29}$. Adicionalmente, promueven un aumento en los niveles de glutatión en los tejidos, elevación de la capacidad antioxidante del plasma ${ }^{27}$ y una mayor biogénesis mitocondrial ${ }^{29}$, permitiendo una mejora en la defensa antioxidante y disminuyendo los niveles de biomarcadores de estrés oxidativo ${ }^{30}$.

\section{Efectos de distintos protocolos de ejercicio físico sobre marcadores de estrés oxidativo en pacientes con diabetes mellitus tipo 2}

Para el tratamiento de la DMT2, los principales protocolos de ejercicio físico recomendados son los ejercicios continuos de moderada intensidad $(\mathrm{ECMI})^{31}$, ejercicios de sobrecarga $(\mathrm{ES})^{32}$, ejercicios intervalados de alta intensidad (EIAI) ${ }^{33}$, y la combinación de ECMI y ES conocida como ejercicio concurrente $(\mathrm{EC})^{34}$.

\subsection{Ejercicio de sobrecarga}

El ejercicio de sobrecarga (ES), también conocido como "ejercicio de fuerza" o de "contra resistencia", consiste en la ejecución de movimientos monoarticulares o poliarticulares en contra de una resistencia, la cual generalmente es propiciada por pesos libres o máquinas de sobrecarga ${ }^{32}$.

Aunque la evidencia muestra que la realización de ES promueve una mejora de la salud metabólica en pacientes con $\mathrm{DMT}^{35}$, no se han observado 
cambios significativos sobre parámetros de EO en este tipo de pacientes ${ }^{36}$. Interesantemente, la respuesta del ES sobre marcadores de EO parece depender del estado de salud de los pacientes, observándose una disminución en los niveles plasmáticos de $\mathrm{MDA}^{37,38}$ y un aumento en la actividad sanguínea de GPx en sujetos sanos posterior a protocolos de 3 series a $65-70 \%$ de 1 RM, 3 veces por semana durante 6-8 semanas ${ }^{37}$. Así mismo, posterior a protocolos de alta intensidad ( 3 x 3-6 repeticiones a $85-90 \%$ de $1 \mathrm{RM}$ ) se ha constatado un aumento de la actividad de SOD en sangre ${ }^{37}$. Sin embargo, una diferencia importante entre los trabajos con sujetos sanos ${ }^{37,38}$ y el ensayo con pacientes diabéticos ${ }^{36}$ es la diferencia de edades entre los participantes, siendo los primeros menores a 30 años, mientras que los segundos son mayores a 50 años. Por lo tanto, dada la escasa información existente en relación al ES y el estrés oxidativo en pacientes con DMT2, no es posible identificar si la baja efectividad de este protocolo se debe a una menor respuesta de adaptación propia de los pacientes con DMT2 o si depende de la edad de los sujetos (Tabla 2 ).

\subsection{Ejercicio continuo de moderada intensidad}

El ejercicio continuo de moderada intensidad (ECMI) o "ejercicio aeróbico" es un tipo de ejercicio caracterizado por la predominancia de la vía metabólica oxidativa y por la ejecución de modalidades cíclicas (caminata, trote, bicicleta, etc.) que involucran grandes grupos musculares ${ }^{39}$. Según la metodología empleada, la intensidad del ejercicio puede ser baja o moderada, siendo esta última fundamental en la regulación y control de la glicemia ${ }^{39}$.

Respecto a los efectos del ECMI sobre biomarcadores relacionados al estrés oxidativo en pacientes con DMT2, en la mayoría de los ensayos se han observado mejoras en relación al balance redox, tanto por un aumento de los niveles de biomarcadores antioxidantes en sangre e $^{37,40-42}$ como por una disminución de biomarcadores de oxidación de proteínas en sangre ${ }^{42}$ y ADN en orina $^{43}$. Así mismo, dichas respuestas coinciden con efectos positivos sobre diversos parámetros clínicos tales como incremento de la función cardiovascular ${ }^{36,40-42}$, control del perfil lipídico ${ }^{37}$, mejora de la composición corporal ${ }^{41,43}$ y control glicémico ${ }^{41,43}$. Solo en un estudio no se hallaron cambios estadísticamente significativos en la abundancia/actividad antioxidante ni en marcadores de daño por estrés oxidativo, específicamente en los niveles de $\mathrm{MDA}^{33}$. A pesar de esto, se observaron efectos positivos sobre parámetros clínicos como glicemia en ayunas, índice HOMA y el porcentaje de grasa corporal ${ }^{33}$. Interesantemente, Krause y colaboradores reportaron un aumento significativo en la actividad de la CAT en sangre posterior a un protocolo de caminata libre a moderada intensidad, y una disminución de los niveles de proteínas carboniladas con protocolos de baja/moderada intensidad. Sin embargo, dichos efectos ocurrieron en ausencia de cambios estadísticamente significativos en parámetros como el porcentaje de grasa corporal, glicemia en ayunas o índice $\mathrm{HOMA}^{42}$. No obstante, sí se observaron cambios clínicamente significativos en el porcentaje de grasa corporal, constatándose disminuciones de aproximadamente $2 \%$ posterior a la intervención ${ }^{42}$. Dado que la mayoría de los protocolos emplean tiempos de ejercicio iguales o superiores a 30 min e intensidad moderada, la respuesta al ECMI sobre los niveles de EO parece ser dependiente de la duración de la intervención y la edad de los sujetos, mientras que la modalidad de ejercicio empleada no parece influir. De este modo, se observa que en sujetos con una edad promedio $61,7 \pm 2,7$ años se requiere de un tiempo de intervención igual o superior a las 16 semanas, mientras que, en grupos menores a 60 años, 12 semanas parecen ser suficientes para constatar cambios (Tabla 2).

\subsection{Ejercicio intervalado de alta intensidad}

El ejercicio intervalado de alta intensidad (EIAI), también conocido por su sigla en inglés "HIIT" ("high-intensity interval training") o "ejercicio intermitente", se caracteriza por la realización reiterada de breves períodos de trabajo (de $10 \mathrm{seg}$ hasta $4 \mathrm{~min}$ ) a una intensidad relativa superior al umbral anaeróbico, seguido de periodos de recuperación a baja intensidad o descanso absoluto ${ }^{44}$.

Respecto a los efectos del EIAI sobre marcadores de estrés oxidativo en pacientes con DMT2, la evidencia es escasa. Un ensayo clínico realizado por Mitranun y colaboradores ${ }^{33}$ reclutó a 43 pacientes, los cuales fueron distribuidos aleatoriamente en tres grupos: I) EIAI, II) ECMI y III) grupo control que permaneció sin realizar ejercicio. La intervención de entrenamiento consistió en trotar sobre un tapiz rodante durante 12 


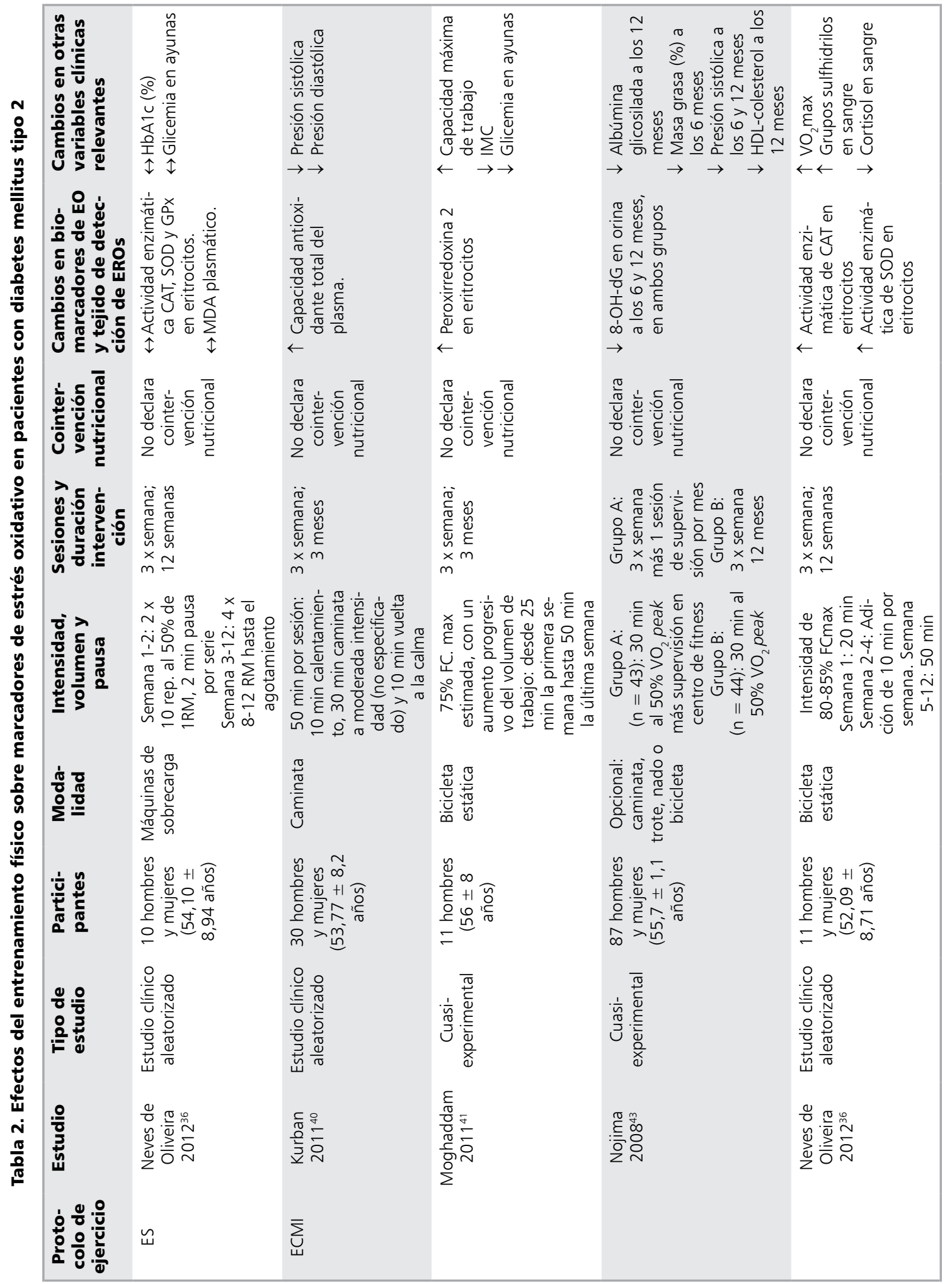




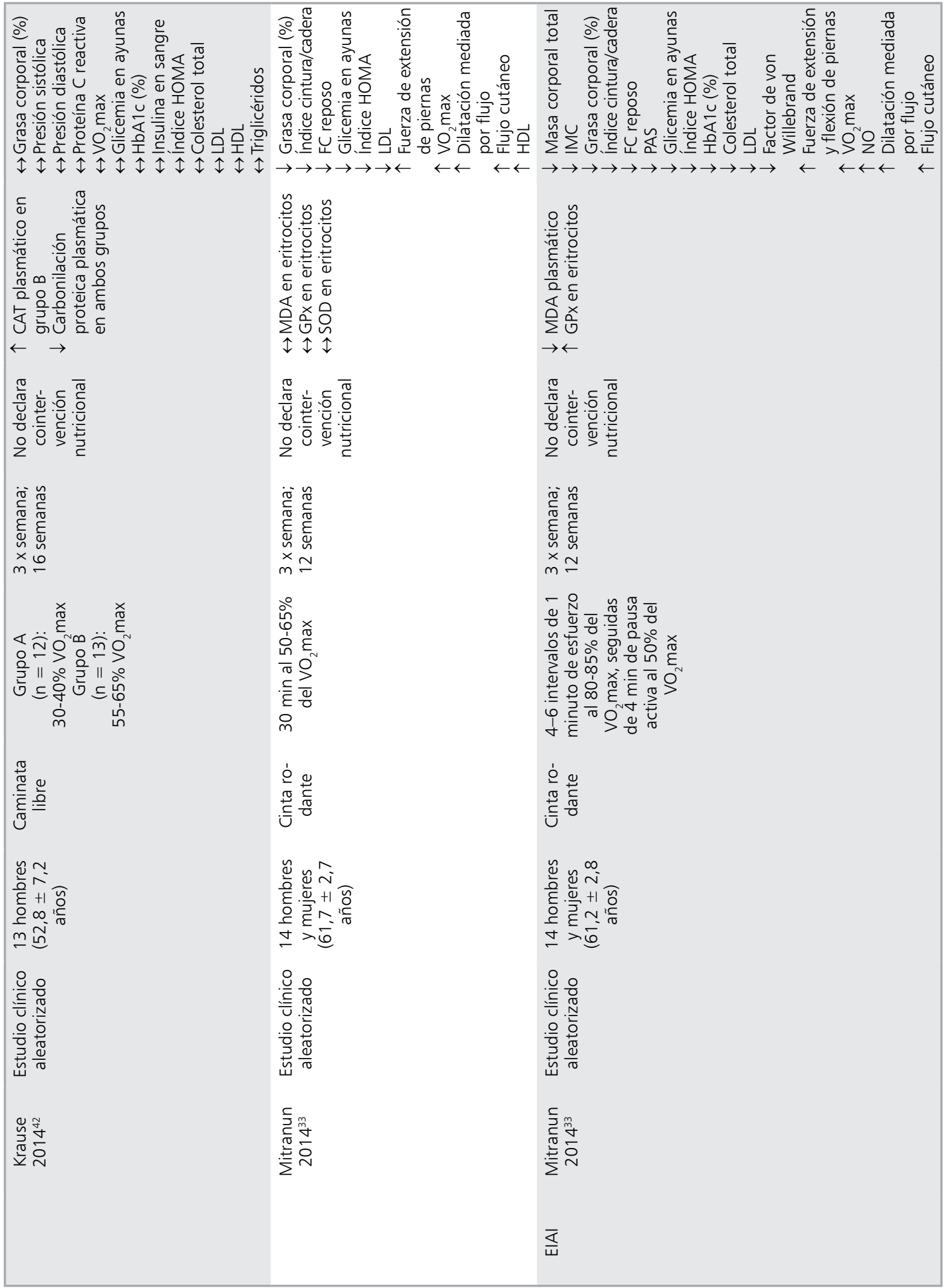




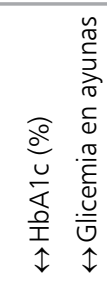

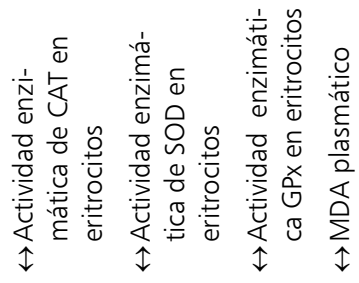

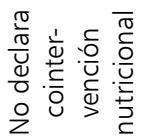

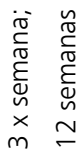

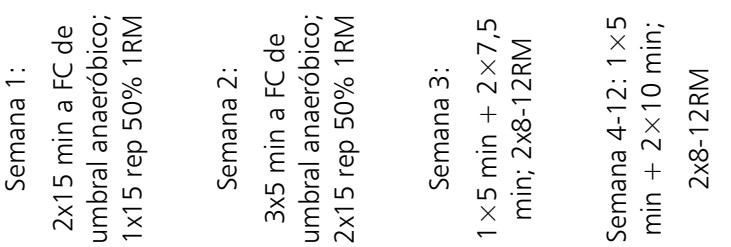

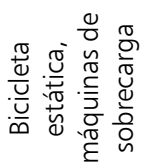

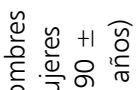

을

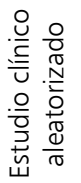

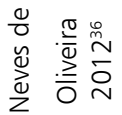

$\breve{u}$

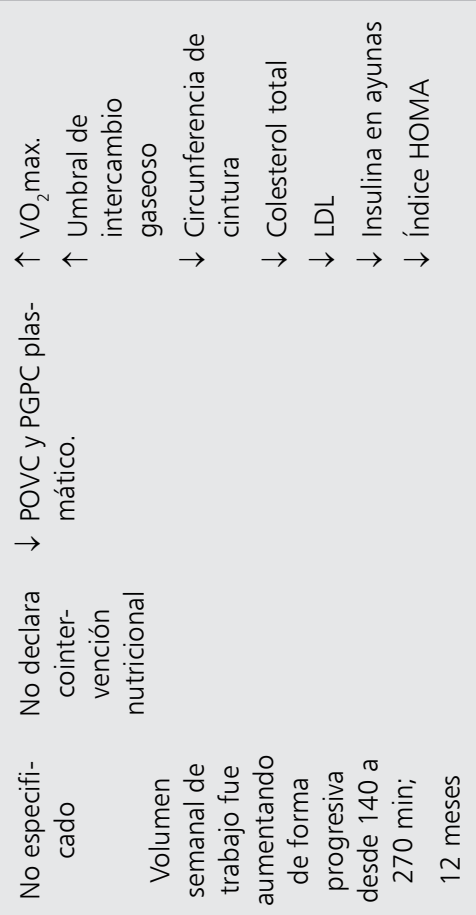

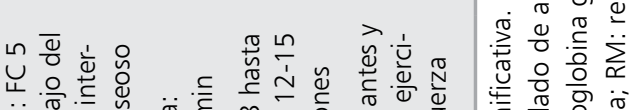

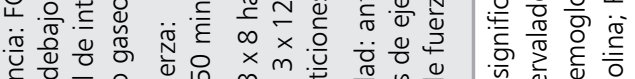

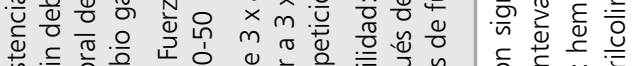

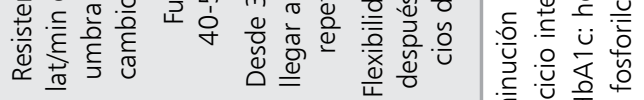

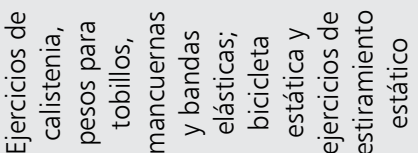

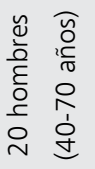

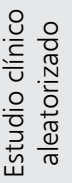

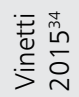
हो

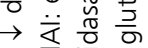

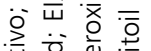

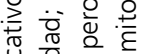
는 $\simeq \frac{1}{\sqrt{0}}$

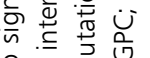
응 긍

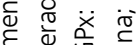
उ.

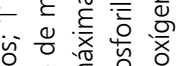

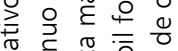

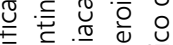

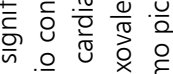

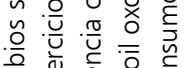

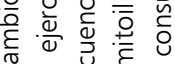

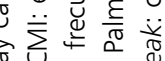

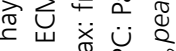
월 है

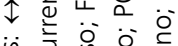

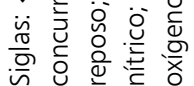


semanas con tres sesiones por semana, con un aumento en la duración de la sesión desde 30 a 60 min. Si bien no se observaron cambios estadísticamente significativos en las concentraciones de SOD en ninguno de los grupos experimentales, sólo en el grupo de EIAI se observó una disminución significativa de MDA y un aumento de la actividad de la enzima $\mathrm{GPX}^{33}$. Estos resultados coincidieron con una disminución de \%HbAlc, glicemia en ayunas e índice HOMA, además de la mejora de otros parámetros clínicos relacionados con la función cardiovascular, perfil lipídico y composición corporal ${ }^{33}$ (Tabla 2).

\subsection{Ejercicio concurrente}

El protocolo de ejercicio concurrente (EC) generalmente combina el ECMI con ES dentro de la misma sesión de entrenamiento ${ }^{45}$. A nivel metabólico se han demostrado sus beneficios sobre el control glicémico en pacientes con DMT2 ${ }^{46}$. Respecto a la efectividad del EC sobre parámetros de EO se ha observado que dichos efectos parecen ser evidentes a largo plazo. Neves de Oliveira y colaboradores no hallaron cambios significativos en la actividad antioxidante de GPx, SOD, CAT ni en los niveles de MDA en sangre posterior a un protocolo de 12 semanas de duración ${ }^{36}$. Por otra parte, Vinetti y colaboradores, quienes aplicaron un protocolo de ejercicios de 12 meses, observaron una disminución significativa en marcadores de daño de la membrana plasmática inducidos por EO, además de una disminución del índice HOMA y una mejora significativa de la composición corporal, el perfil lipídico y la función cardiovascular en pacientes con DMT2 ${ }^{34}$ (Tabla 2).

\section{Conclusiones}

El ejercicio físico es un medio fundamental para la prevención y tratamiento de diversas enfermedades crónicas como la DMT2. A su vez, una óptima prescripción de ejercicio físico es capaz de inducir respuestas de adaptación que conducen a la mantención del balance redox al interior del organismo. Considerando lo anterior y el papel que desempeña el EO en la patogénesis de la DMT2 y el desarrollo de complicaciones asociadas a la enfermedad, el ejercicio físico parece ofrecer nuevos beneficios para el control de pacientes con DMT2.
La evidencia encontrada y resumida en la presente revisión sugiere que la efectividad del ejercicio físico en la disminución de biomarcadores de EO depende principalmente del protocolo de ejercicios aplicado a los pacientes con DMT2. De este modo, los protocolos ECMI y EIAI parecen ser estrategias de mayor efectividad, mientras que el ES y el EC han mostrado tener una menor evidencia respecto a su eficacia en la disminución de los biomarcadores de estrés oxidativo. No obstante, la mayoría de los protocolos de ejercicio estudiados presentan mejoras significativas en parámetros clínicos tales como el control glicémico, composición corporal y la función cardiovascular. A futuro, se requieren nuevos estudios que indaguen la relevancia de los efectos del ejercicio sobre el balance redox en el contexto de la prevención de enfermedades asociadas al EO crónico.

\section{Referencias}

1. Ministerio de Salud de Chile. Encuesta Nacional de Salud 2009-2010. Disponible:http://web.minsal.cl/portal/ url/item/bcb03d7bc28b64dfe040010165012d23.pdf [Consultado el 13 de marzo de 2017).

2. DeFronzo R, Ferrannini E, Groop L, Henry R, Herman W, Juul Holst J, et al. Diabetes Mellitus type 2. Nat Rev Dis Primers 2015; 1: 15019.

3. Johnson CB, Davis MK, Law A, Sulpher J. Shared Risk Factors in Cardiovascular Disease and Cancer Implications for Preventive Health and Clinical Care in Oncology Patients. Circulation 2016; 32 (7): 900-7.

4. Frijhoff J, Winyard PG, Zarkovic N, Davies SS, Stocker $\mathrm{R}$, Cheng D, et al. Clinical Relevance of Biomarkers of Oxidative Stress. Antioxid Redox Signal 2015; 23 (14): 1144-70.

5. Henriksen EJ, Diamond-Stanic MK, Marchionne EM. Oxidative stress and the etiology of insulin resistance and type 2 diabetes. Free Radic Biol Med 2011; 51 (5): 993-9.

6. Giacco F, Brownlee M. Oxidative stress and diabetic complications. Circulation Research 2010; 107 (9): 1058-70.

7. Pareja-Galeano H, Garatachea N, Lucia A. Exercise as a Polypill for Chronic Diseases. Molecular and Cellular Regulation of Adaptation to Exercise 2015; 135: 497526.

8. Pedersen BK, Saltin B. Exercise as medicine-evidence for prescribing exercise as therapy in 26 different chronic diseases. Scand J Med Sci Sports 2015; 25 (S3): 1-72. 
9. Umpierre D, Ribeiro P, Kramer C, Leitao C, Zucatti A, Azevedo M, et al. Physical activity advice only or structured exercise training and association with $\mathrm{HbAlc}$ levels in type 2 diabetes: a systematic review and meta-analysis. J Am Med Assoc 2011; 305 (17): 1790-9.

10. Thomas D, Elliott E, Naughton G a. Exercise for type 2 diabetes mellitus. Cochrane Database Syst Rev 2006; 3 (1): CD002968.

11. Powers S, Radak Z, Ji LL. Exercise-induced oxidative stress: past, present and future. J Physiol 2016; 594 (18): 5081-92.

12. Pisoschi AM, Pop A. The role of antioxidants in the chemistry of oxidative stress: A review. Eur J Med Chem 2015; 97: 55-74.

13. Toro J, Rodrigo R. Oxidative Stress: Basic Overview. En: Oxidative Stress and Antioxidants: Their Role in Human Disease. New York, United States of America: Nova; 2009. p. 1-24.

14. Rodrigo R, Brito R, González-Montero J, Benedetti V. Antioxidants in human disease: Potential therapeutic opportunities. Clin Pharmacol Transl Med 2017; 1 (2): 44-53.

15. Powers SK, Jackson MJ. Exercise-induced oxidative stress: cellular mechanisms and impact on muscle force production. Physiol Rev 2008; 88 (4): 1243-76.

16. Mañon W, Garrido G, Núñez A. Biomarcadores del estrés oxidativo en la terapia antioxidante. Journal of Pharmacy \& Pharmacognosy Research 2016; 4 (2): 6283.

17. Saben J, Boudoures A, Asghar Z, Thompson A, Drury A, Zhang W, et al. Maternal metabolic syndrome programs mitochondrial dysfunction via germline changes across three generations. Cell Rep 2016; 16 (1): 1-8.

18. Padron de Figueiredo A, Salmon A, Bruno F, Jiménez F, Martínez H, Halade G, et al. Nox2 Mediates Skeletal Muscle Insulin Resistance Induced by a High Fat Diet. The Journal of Biological Chemistry 2015; 290: 1342739.

19. Tangvarasittichai S. Oxidative stress, insulin resistance, dyslipidemia and type 2 diabetes mellitus. World J Diabetes. Baishideng Publishing Group Inc 2015; 6 (3): 456-80.

20. Kaneto H, Katakami N, Matsuhisa M, Matsuoka T. Role of Reactive Oxygen Species in the Progression of Type 2 Diabetes and Atherosclerosis. Mediators Inflamm 2010; 2010: 1-11.

21. Olivares Reyes J, Arellano Plancarte A. Bases moleculares de las acciones de la Insulina. Rev Educ Bioquímica 2008; 27 (1): 9-18.

22. Le Lay S, Simard G, Martínez MC, Andriantsitohaina R. Oxidative stress and metabolic pathologies: from an adipocentric point of view. Oxid Med Cell Longev 2014; 2014: 908539.

23. Wright E, Scism-Bacon JL, Glass LC, Glass L. Oxidative stress in type 2 diabetes: the role of fasting and postprandial glycaemia. Int J Clin Pract 2006; 60 (3): 308-14.

24. Nowotny K, Jung T, Höhn A, Weber D, Grune T. Advanced glycation end products and oxidative stress in type 2 diabetes mellitus. Biomolecules Multidisciplinary Digital Publishing Institute (MDPI) 2015; 5 (1): 194 222.

25. Jackson MJ, Vasilaki A, McArdle A. Cellular mechanis$\mathrm{ms}$ underlying oxidative stress in human exercise. Free Radic Biol Med 2016; 98: 13-7.

26. Díaz-Vegas A, Campos C, Contreras-Ferrat A, Casas M, Buvinic S, Jaimovich E, et al. ROS Production via P2Y1-PKC-NOX2 Is Triggered by Extracellular ATP after Electrical Stimulation of Skeletal Muscle Cells. Plos One 2015; 10 (6): e0129882.

27. Bogdanis G, Stavrinou P, Fatouros I, Philippou A, Chatzinikolaou A, Draganidis D, et al. Short-term high-intensity interval exercise training attenuates oxidative stress responses and improves antioxidant status in healthy humans. Food and Chemical Toxicology 2013; 61: 171-7.

28. Rodrigo R, Prieto J, Castillo R. Cardioprotection against ischaemia/reperfusion by vitamins $C$ and $E$ plus $n-3$ fatty acids: molecular mechanisms and potential clinical applications. Clinical Science 2013; 124: 1-15.

29. Ji LL, Kang C, Zhang Y. Exercise-induced hormesis and skeletal muscle health. Free Radic Biol Med 2016; 98: 113-22.

30. Ji LL, Adams V, Nehrhoff B, Spate U, Linke A, Schulze $\mathrm{P}$, et al. Redox signaling in skeletal muscle: role of aging and exercise. Adv Physiol Educ 2015; 39 (4): 352-9.

31. Gómez-Cabrera M, Domenech E, Viña J. Moderate exercise is an antioxidant: Upregulation of antioxidant genes by training 2008; 44 (2): 126-31.

32. Gacitua T, Karachon L, Romero E, Parra P, Poblete C, Russell J, et al. Effects of resistance training on oxidative stress-related biomarkers in metabolic diseases: a review. Sport Sci Health 2017. Disponible: https://link.springer. com/article/10.1007/s11332-017-0402-5\#citeas [Consultado el 15 de octubre de 2017).

33. Mitranun W, Deerochanawong C, Tanaka H, Suksom D. Continuous vs interval training on glycemic control and macro- and microvascular reactivity in type 2 diabetic patients. Scand J Med Sci Sport 2014; 24 (2): 69-76.

34. Vinetti G, Mozzini C, Desenzani P, Boni E, Bulla L, Lorenzetti I, et al. Supervised exercise training reduces 
oxidative stress and cardiometabolic risk in adults with type 2 diabetes: a randomized controlled trial. Sci Rep 2015; 5: 9238.

35. Gordon BA, Benson AC, Bird SR, Fraser SF. Resistance training improves metabolic health in type 2 diabetes: A systematic review. Diabetes Res Clin Pract 2009; 83 (2): 157-75.

36. Neves de Oliveira V, Bessa A, Mendonça Pereira JML, Da Silva Oliveira RJ, de Mello MT, De Agostini GG, et al. The effect of different training programs on antioxidant status, oxidative stress, and metabolic control in type 2 diabetes. Appl Physiol Nutr Metab 2012; 37 (2): 334-44.

37. Azizbeigi K, Azarbayjani MA, Atashak S, Stannard SR. Effect of moderate and high resistance training intensity on indices of inflammatory and oxidative stress. Res Sports Med 2015; 23 (1): 73-87.

38. Cakir-Atabek H, Demir S, PinarbaŞili RD, Gündüz N. Effects of different resistance training intensity on indices of oxidative stress. J Strength Cond Res 2010; 24 (9): 2491-7.

39. Quílez Llopiz P, Reig García-Galbis M. Glycemic control through physical exercise in type 2 diabetes systematic review. Nutr Hosp 2015; 31 (4): 1465-72.

40. Kurban S, Mehmetoglu I, Yerlikaya HF, Gonen S, Erdem S. Effect of chronic regular exercise on serum ischemia-modified albumina levels and oxidative stress in type 2 diabetes mellitus. Endocr Res 2011; 36 (3): 116-23.

41. Moghaddam DA, Heber A, Capin D, Kreutz T, Opitz $\mathrm{D}$, Lenzen $\mathrm{E}$, et al. Training increases peroxiredoxin 2 contents in the erythrocytes of overweight/obese men suffering from type 2 diabetes. Wiener Medizinische Wochenschrift 2011; 161 (21-22): 511-8.

42. Krause M, Rodrigues-Krause J, O’Hagan C, Medlow P, Davison G, Susta D, et al. The effects of aerobic exercise training at two different intensities in obesity and type 2 diabetes: Implications for oxidative stress, low-grade inflammation and nitric oxide production. Eur J Appl Physiol 2014; 114 (2): 251-60.

43. Nojima H, Watanabe H, Yamane K, Kitahara Y, Sekikawa K, Yamamoto $\mathrm{H}$, et al. Effect of aerobic exercise training on oxidative stress in patients with type 2 diabetes mellitus. Metabolism 2008; 57 (2): 170-6.

44. Buchheit M, Laursen PB. High-intensity interval training, solutions to the programming puzzle: Part I: cardiopulmonary emphasis. Sports Med 2013; 43 (5): 313-38.

45. Coffey VG, Hawley JA. Concurrent exercise training: do opposites distract? J Physiol 2016; 595 (9): 2883-96.

46. Oliveira C, Simões M, Carvalho J, Ribeiro J. Combined exercise for people with type 2 diabetes mellitus: a systematic review. Diabetes Res Clin Pract 2012; 98 (2): 187-98. 